\title{
Medical Futility in Cancer Care: Distinct Challenges and Action Strategies
}

\section{Gallagher $\mathrm{CM}^{*}$}

Integrated Ethics in Cancer Care; The University of Texas MD Anderson Cancer Center, USA

*Corresponding author: Colleen M Gallagher, Integrated Ethics in Cancer Care, The University of Texas MD Anderson Cancer Center, 1400 Pressler Street-Unit 1430 Houston, TX 77030, USA, Tel: 713-792-8775; Work: 713-792-3204; FAX: 713-745-0674; E-mail: CMGallagher@mdanderson.org

Received date: February 26, 2016; Accepted date: April 01, 2016; Published date: April 04, 2016

Copyright: ( 2016 Gallagher CM. This is an open-access article distributed under the terms of the Creative Commons Attribution License, which permits unrestricted use, distribution, and reproduction in any medium, provided the original author and source are credited.

\begin{abstract}
Clinical cases involving the concern of medical futility can be especially challenging within the context of cancer care. We offer two considerations of which one should be mindful when dealing with cases involving medical futility; neither have been extensively discussed in the existing literature. Then, we explain how these considerations can become even more pronounced within a cancer-care setting, and present action strategies for clinicians and clinical ethicists who confront these issues within the clinical environment. The goal of these action strategies is to assist clinicians and clinical ethicists in communicating effectively with patients and their families about situations involving requests for medically futile interventions.
\end{abstract}

Keywords: Medical futility; Cancer care; Ethicists; Clinical environment

\section{Introduction}

It is frequently claimed that physicians have an ethical obligation to deny requests for interventions that are medically futile, and to avoid presenting such options to patients and their families as treatment possibilities [1-5]. This claim has also been questioned by those who regard the notion of medical futility as inadequately characterized, such that any decision to withhold treatment based on considerations of futility would be highly suspect [5-10]. Despite this disagreement, it is possible to glean from the literature a distinction between medical futility, characterized in terms of empirically predictable clinical efficacy of an intervention relative to a particular goal, and other notions of futility, which are overtly value-laden.

The most frequently cited account of futility characterizes this concept in the following way:

"A futile action is one that cannot achieve the goals of the action, no matter how often repeated. The likelihood of failure may be predictable because it is inherent in the nature of the action proposed, and it may become immediately obvious or may become apparent only after many failed attempts. Futility refers to an expectation of success that is either predictably or empirically so unlikely that its exact probability is often incalculable" [3].

The notion of futility described here is sometimes referred to as a quantitative notion of futility, because it characterizes futile action in terms of quantifiable probability or likelihood of failure $[3,11]$. There are subtle differences that exist between various characterizations of quantitative futility, but for our purposes, to say that an intervention is quantitatively futile is to say that it is exceedingly unlikely to accomplish its intended clinical goal. This conceptualization of futility is markedly different from what is sometimes referred to as a qualitative notion of futility, whereby one determines that a particular end is of no value, and that interventions which aim at achieving that end are therefore medically futile [5,11]. For example, one might regard mechanical ventilation in a critically ill patient with little hope of recovery as medically futile, because the end of prolonging the patient's life under such conditions is regarded as having no value.

Unlike quantitative futility, determining that an intervention is qualitatively futile requires that one makes a normative judgment about the value of a particular end. Many factors will contribute to this determination but at base, the team will seek out information that places the current medical situation within the larger context of the person's lived life. In this way, an intervention's futility is extremely variable. This judgment, importantly, may be at odds with the patient's own perspective regarding the value of this end, which can lead to conflict about whether an intervention is truly medically futile. Qualitative notions of futility are frequently criticized for precisely this reason. While it is easy to see why a physician may be obligated to refuse to provide quantitatively futile interventions, it is less clear that the same obligations hold in cases of qualitative futility (Table 1).

\begin{tabular}{|l|l|}
\hline $\begin{array}{l}\text { Gallagher and Holmes Strategies for Discussion with Families or } \\
\text { Surrogate Decision-Makers }\end{array}$ \\
\hline 1. $\quad$ Clarify Goals of Care \\
\hline 2. $\quad$ Assess whether all reasonable options have been attempted \\
\hline 3. $\quad$ Do not offer options that are not medically appropriate \\
\hline $4 . \quad$ Establish guidelines and limits for options in place \\
\hline $5 . \quad$ Seek to address emotional needs of caregivers \\
\hline
\end{tabular}

Table 1: Gallagher and Holmes Strategies.

Ethicists Gallagher and Holmes have offered several suggestions, to both clinicians and clinical ethicists, for how to deal with situations that involve medical futility. They propose that clarifying goals of care, assessing the availability of reasonable treatment options, avoiding offering interventions that are medically inappropriate, establishing guidelines and limits for current interventions, and addressing the emotional needs of patients' caregivers are all strategies that can help in such circumstances [12,13]. They also identify patient care 
conferences, reviewing cases with a colleague, and requesting ethics consults as practical methods for implementing these strategies $[12,13]$.

We agree that these are helpful suggestions, and that utilizing them effectively can help clinicians and clinical ethicists to work through cases of medical futility successfully. However, we propose that there are at least two additional considerations that ought to be incorporated into one's ethical analysis of medical futility cases.

First, determinations of medical futility in a given clinical situation ought to specify whether the intervention in question is one of quantitative or qualitative futility. These concepts are frequently conflated in the clinical environment, but they have different ethical significance, which is brought to bear on the sorts of strategies that are appropriate in each type of case. Second, we claim that in cases of quantitative futility, the institutional and provider-based preconceptions involved in making determinations of quantitative futility should be explicitly discussed. While quantitative futility is more empirically grounded than qualitative futility, there remains an element of clinical judgment in determinations of quantitative futility in at least some cases. For example, sometimes clinicians may order an intervention based on their own clinical experience, which is not supported by empirical evidence. This is not to say that there is evidence against such an intervention, but rather that there is a lack of empirical data in either direction. In the absence of a generalizable base of evidence, years of practice and associated experience become the individualized store of data that is used to inform such determinations. Other clinicians, however, may regard that same intervention as medically futile, based on a different set of clinical experiences. So while quantitative futility does not involve normative judgments, as we saw in the case of qualitative futility, determinations of quantitative futility sometimes do involve clinical judgments based on individualized experience, rather than or in addition to empirical data.

\section{Two Considerations Regarding Medical Futility Cases}

In addressing questions of medical futility, we propose that there are two additional considerations not currently discussed in the existing literature, which may be helpful. First, quantitative and qualitative futility are easily conflated within the clinical environment. Consider the following case:

D.T. is a critically ill patient with Stage 4 lung cancer. D.T. is in multi-organ failure in the I.C.U., and her physicians are considering using vasopressors to correct her newly onset hypotension. D.T.s attending physician, however, is concerned that vasopressors are medically futile in this case, in light of D.T.s poor prognosis. Vasopressors will not move the patient toward the goal of safely discharging from the hospital, nor will they correct any of her most serious underlying issues. D.T.s physician is concerned that if vasopressors are a medically futile intervention, he is under an obligation to refrain from offering them as a treatment option for D.T.

D.T.s physician seems to make a good point-vasopressors will not help resolve D.T.s underlying medical issues. At most, they may extend her life for a short period of time, but in all likelihood D.T. will die in the hospital whether she receives vasopressors or not. Insofar as one can predict, with a reasonable degree of certainty, that vasopressors will not advance the goal of allowing D.T. to discharge safely from the hospital, vasopressors are medically futile in a quantitative sense in this case. But one might also come to a determination of qualitative futility in this case. Suppose D.T.s attending physician determines that the goal of extending her life for a short period, which he acknowledges to be the most likely consequence of giving vasopressors to D.T., is not a goal worth pursuing. Thus, the attending physician regards vasopressors as qualitatively futile in this case as well.

This case helps to highlight the subtle but ethically significant difference between determinations of quantitative versus qualitative futility. The judgment that vasopressors are quantitatively futile, relative to the goal of discharging safely from the hospital, is based on an assessment of the clinical facts. Given D.T.s prognosis and current condition, vasopressors are almost certain to be ineffective in moving D.T. toward the goal of discharge from the hospital. Coming to this conclusion does not require that D.T.s physician make a normative judgment about the value of this goal. However, the judgment that vasopressors are qualitatively futile does require that D.T.s physician make a normative judgment about the value of a particular goal. The determination that in this case, vasopressors are qualitatively futile requires the judgment that the goal of extending D.T.'s life for a short period of time is not a goal worth pursuing.

From this, one can recognize why the difference between qualitative and quantitative futility is ethically significant. As we noted earlier, it is often held that physicians have an ethical obligation to deny requests for interventions that are medically futile, and to avoid presenting such options to patients and their families as treatment possibilities. Critics of this claim have argued that medical futility is too inherently valueladen to give rise to an ethical obligation of this type, but this criticism does not apply to determinations of quantitative futility. Determinations of quantitative futility are based on clinical judgments about the probable effectiveness of a given intervention, supported by empirical data when available, toward achieving a specified goal, without regard for whether that goal is of value. The ethical obligation to deny requests and avoid offering interventions which are quantitatively futile is effectively an obligation to withhold interventions that are exceedingly unlikely to work. While one may take issue with the notion that qualitative futility gives rise to such an obligation, it is difficult to deny the claim that physicians should not provide interventions that will be clinically ineffective.

One can also recognize from this example why cases involving questions of medical futility can give rise to conflicts between patients and their families. For example, suppose that D.T.s family understands her prognosis, and is hoping to travel from out-of-state so that they can be at her bedside when she dies. In this case, the use of vasopressors to control D.T.s hypotension is not quantitatively futile, relative to the goal of prolonging D.T.s life for a short time. This determination is in conflict with the judgment of the physician, however, who arrives at a judgment of quantitative futility based on assessing the effectiveness of vasopressors relative to the goal of safe discharge from the hospital. In this case, a disagreement about quantitative futility emerges because each party is assessing the predictable utility of the intervention in light of different goals. An intervention may be more effective in one respect, but less effective in another, which can give rise to disagreements about whether an intervention is quantitatively futile.

Disagreement regarding qualitative futility is also possible in this case. For D.T.s family, and perhaps for D.T. as well, the goal of sustaining D.T.s life for a short period of time is of significant value, because it may be important for both D.T. and her family that they are together at the time of her passing. This is in stark contrast with the judgment of the physician, who regards the brief prolonging of D.T.s 
life as a goal not worth pursuing. So while disagreement about quantitative futility emerges when parties evaluate the efficacy of an intervention relative to different goals, disagreement about qualitative futility arises when parties disagree about the value of the same goal. This can make disagreements about medical futility in a particular case extremely complicated to resolve.

Next, let us consider the role of institutional- and provider-specific biases in determinations of quantitative futility. For example, suppose that in the above case, D.T.s oncologist determines that vasopressors will advance the goal of allowing D.T. to discharge safely from the hospital, albeit indirectly, because they believe that extending D.T.s life for a short period of time may allow for D.T. to receive additional cancer therapies. In the oncologist's view, vasopressors are not quantitatively futile in D.T.s case, even relative to the goal of discharging safely from the hospital. This is in sharp contrast to the view held by D.T.s attending ICU physician, who regards vasopressors as wholly ineffective in advancing the goal of safe discharge from the hospital for D.T. Each provider brings their own clinical experiences, and professional outlook, to the table in assessing whether vasopressors are quantitatively futile in D.T.s case, which leads to differing determinations of quantitative futility. These sorts of provider-specific leanings can meaningfully influence whether a particular provider regards an intervention as quantitatively futile in a specific case.

Now suppose that D.T.s oncologist is practicing at an institution with significant investment in research on investigational therapies, including Phase I clinical research. They may regard the use of vasopressors for the purpose of extending D.T.s life as an opportunity to not only offer D.T. the possibility, however slight, of benefitting from additional therapies still being researched, but also to advance the goal of developing novel cancer therapies. An oncologist in a researchoriented institution may regard certain interventions as effective relative to research goals that are not within the purview of practitioners at institutions that lack such orientations. Thus, even within the same clinical specialization, there may be institutional preferences that lead to differing determinations of quantitative futility in a given case.

\section{Medical Futility in a Cancer Care Setting: Three Additional Challenges}

There are at least three features of cancer care that make cases of medical futility especially challenging, and to which the above considerations are particularly pertinent. First, cancer-care patients tend to be willing to pursue courses of treatment for which there is very little direct evidence of efficacy. For example, this patient population tends to be willing to participate in Phase I clinical trials, which involve study drugs for which there is usually little direct evidence of effectiveness in humans [14-16]. As with all studies involving human test subjects, regulatory bodies such as institutional review boards (IRBs) or ethics committees as they are sometimes called in non-US contexts, are obligated to judiciously assess proposed research with a particular focus on protecting the patient-subjects who will be enrolled. While such bodies serve a critical function regardless of the agent or intervention being investigated, the potentially highstakes nature of first-in-human studies underscores the importance of the review process as Phase I trials are intended to evaluate drug safety, determine safe dosage limitations, and identify side effects [17]. As such, Phase I trials are technically not intended to evaluate efficacy at all. Despite objective success rates of less than 5\%, numerous cancer patients elect to participate in Phase I clinical trials, and they often do so with the expectation, or at least the hope, that they will receive a curative benefit $[14,18,19]$. For this population, even minimal evidence of effectiveness is seen as adequate to justify pursuing further treatment. This can make cancer patients less likely to regard any intervention as quantitatively futile, because they are accustomed to pursuing treatments for which the likelihood of benefit is exceedingly small.

Another feature of cancer care that tends to make cases of medical futility especially challenging has to do with the prominent role of clinical trials in cutting-edge cancer care. Many cancer-care institutions have a significant research agenda, and a strong commitment to developing novel cancer therapies. This work depends somewhat crucially on patients' willingness to participate in clinical trials, including Phase I first-in-human studies, but it also depends on the willingness of physicians to recruit participants to enroll in clinical trials of these drugs. This is not to say that physicians who practice in research-oriented institutions have no choice in the matter; it is simply a routinely considered option if one is involved in cutting-edge cancer research. Thus, physicians working in a cancer institution that conducts research must also, at some level, be committed to an exceedingly low empirical threshold for determining the potential utility of an available treatment. Beyond a novel use scenario, only institutions that maintain a robust research program are able to continue providing not only standard therapies, but also providing care that is still in the initial stages of research. Patients at such clinical centers have the potential to become simultaneously eligible for either a Phase I clinical trial or hospice referral. In a research-oriented, cancer-care setting, both patient and physician are necessarily committed to attempting interventions whose uses are not well supported by direct empirical evidence. As a result, in such a setting very few interventions may be regarded as quantitatively futile.

These providers face another complicating factor in terms of the potential interruption in the fiduciary relationship that exists between physician and patient. The duties that exist in the scientist-subject relationship are not identical to those forged between patient and physician. As such, concerns have been raised about patients' ability to provide truly informed consent when their treating physician assumes the dual role of researcher [20]. While there is an acceptance that patients will not see themselves as having transformed in some explicit way from being a patient to be a study participant, physician-scientists ought to be keenly aware of the differences that come with a dual role. This is particularly relevant for physicians who are working with longterm patients who are now on clinical protocols. Such interactions are much more common within a cancer-research setting.

Finally, one might be concerned about the evidence base for providing non-cancer related therapies and interventions to patients with advanced cancer [21-23]. For many non-cancer related therapies and interventions, the study populations for evidence-based standards of care are not comprised of cancer patients, and these standards may not be readily generalizable to patients with advanced cancer as a result. The indications for haemoglobin thresholds for red blood cell transfusion in adults provide a useful example. In a 2012 Cochrane Database review, Preston et al. were unable to locate any randomized control trials assessing the utility of transfusion in patients with advanced cancer. The review returned twelve observational studies which identified a variety of subjective responses among patients $(n=653)$ but a significant number of these patients died within two weeks of having received the transfusion (range 23\%-35\%). And yet, 
patients with advanced cancer often receive transfusions in accordance with the same standard of care that is utilized for patients who do not have cancer. Without a comprehensive evidence base, we maintain an inadequate understanding of whether patients with advanced cancer benefit from transfusion and for how long. The predictive power of the biomedical sciences rests on the strength of the evidence, yet in this context, the lack of data must be counter-balanced by anecdotal knowledge. As such, clinical discernment plays a critical role in determining when to provide non-cancer related therapies for patients with advanced cancer, because the standards of care may not be generalizable to people with cancer. This can make the role of provider-specific biases in determinations of quantitative futility even more pronounced. For non-cancer related therapies, there is simply not as much available evidence regarding efficacy among patients with advanced cancer.

The problem of generalizability is not unique to cancer patients. To continue with the haemoglobin example, indications for transfusion based on haemoglobin levels were built on canine studies that were never repeated in humans, much less patients who have been exposed to toxic chemotherapeutics over long periods of time. But the problem of generalizability is decidedly more pronounced among cancer patients. This is exacerbated, perhaps, by the urgency that accompanies a diagnosis of cancer, or because clinical practice assumes that cancer does not significantly effect a person's biochemical responses when compared to those without cancer. Clinicians in a cancer care setting may be asked to evaluate the quantitative futility of a non-cancer related intervention based on evidence that is derived from research on subjects who do not have cancer, because there is simply no other evidence-base from which to make such an evaluation. For many noncancer related treatments and interventions, any determination of quantitative futility will be based on an assumption, right or wrong, that cancer patients will respond similarly to non-cancer patients. Thus, a determination of quantitative futility for a non-cancer intervention in a patient with cancer often rests on uncertain foundations. This creates an element of uncertainty for determinations of quantitative futility among cancer patients that is not present when working with patients who do not have cancer.

\section{Action Strategies for Cases Involving Medical Futility in a Cancer Care Setting}

Thus far, we have noted two considerations, not previously discussed in the literature, which may be helpful for both clinicians and clinical ethicists in addressing cases of medical futility. We have also noted three features of cancer care that can make cases of medical futility especially challenging to resolve. We will conclude by offering three action strategies that target these issues specifically. When combined with Gallagher and Holmes's earlier work, these strategies provide a comprehensive approach to resolving cases of medical futility in a cancer care setting $[12,13]$. After briefly presenting these strategies, we conclude by discussing how our additions can be of use in these sorts of cases.

\section{Be explicit about connections between particular interventions and goals of care, including non-curative goals of care}

As Gallagher and Holmes helpfully note, clarifying goals of care can provide a common ground and shared understanding for all parties involved in a patient's care $[12,13]$. We claim that in cases that involve concerns of medical futility, it's helpful to utilize this shared understanding to determine whether, and if so in what way, a particular intervention is or is not consistent with the goals of care for the patient. This can be especially useful when assessing the potential futility of a non-curative intervention, such as the use of vasopressors in the case of D.T. discussed earlier. This can also be a worthwhile strategy when attempting to identify the medical purposefulness of a research-oriented goal of care. A patient's participation in a Phase I clinical trial may provide useful information that is beneficial to future patients, for example, even if there is no evidence that the trial drug will benefit that particular individual. Relative to a research-oriented goal of care, a Phase I drug is not medically futile, even if there is no evidence of its curative abilities in the patients currently receiving it.

\section{Maintain the distinction between qualitative and quantitative futility}

In both the medical futility literature and the clinical environment, it's common for the term 'medical futility' to be used to refer to both quantitative and qualitative futility. We have already noted some of the differences between these concepts, and explained why this distinction is ethically significant. But maintaining this distinction is also useful for the purpose of collective decision-making between patients, families, and the medical team. For example, in our earlier case of D.T., the ICU attending may be able to resolve his concern about providing vasopressors by reframing this intervention relative to the goal of extending D.T.s life long enough for her family to travel to her bedside. Even if D.T.s physician regards this as a goal not worth pursuing, it doesn't follow that this intervention is therefore futile in the quantitative sense. Similarly, if D.T.s family were to request vasopressors in the hope that they will help advance the goal of safe discharge from the hospital, minding the distinction between quantitative and qualitative futility can help D.T.s physician to properly frame his explanation of why this intervention should not be provided. By being mindful of the fact that in this sort of situation, the relevant sort of futility is quantitative futility, D.T.s physician is able to justify his refusal to provide vasopressors by appealing to their lack of efficacy toward advancing the goal of safe discharge. By maintaining the distinction clarifying the goals of care all people involved in decisionmaking may better understand and agree to goals set. Clarity of expression regarding concerns about medical futility can help patients and families better understand why a physician may be hesitant to offer certain interventions, and it may help physicians to better articulate their concerns about these sorts of cases.

Although the potential utilization of medically futile interventions exists across the continuum of medical care, it might be helpful to address the broader concept of futility within advance-care-planning conversations over the lifecourse. As part of a larger conversation between patient and provider, the idea of medical futility can be introduced without being tied to particular interventions happening at that time. When physicians talked about the utility of antibiotics in the treatment of viral infections, these providers are beginning to challenge patients to think about medical practices along a spectrum with each being marked by predicted usefulness. In these ways, maintaining the distinction between qualitative and quantitative futility in one's presentation and discussion of treatment options can help facilitate a shared understanding of the patient's circumstances, and allow for the possibility of shared decision-making.

Combined Action Strategies 
Page 5 of 6

\begin{tabular}{|l|}
\hline 1. Clarify goals of care \\
\hline $\begin{array}{l}\text { 2. Be explicit about connections between particular interventions and goals of } \\
\text { care, including non-curative goals of care. }\end{array}$ \\
\hline
\end{tabular}

3. Assess whether all reasonable options have been attempted

4. Do not offer options that are not medically appropriate

5. Maintain the distinction between quantitative and qualitative futility

6. Establish guidelines and limits for interventions in place

7. Seek to address the emotional needs of the caregiver

8. Allow for the possibility of reasonable disagreement about whether an intervention is quantitatively futile, and pursue reconciliation of such disagreement on the basis of available facts when possible.

\section{Table 2: Combined Action Strategies.}

\section{Allow for the possibility of reasonable disagreement about whether an intervention is quantitatively futile, and pursue reconciliation of such disagreement on the basis of available facts when possible}

As we discussed previously, in some situations clinicians may disagree about whether an intervention is quantitatively futile in a particular case, based on their differing clinical experiences, clinical specialties, or institutional affiliations. Reasonable, well-supported disagreement among clinicians regarding the quantitative futility of an intervention is not only possible, but expected and tolerable. When such disagreements arise, clinicians should be encouraged to be specific and candid about their reasons for or against a determination of quantitative futility, especially when they are relying on their clinical experience or intuitive judgment, rather than empirically-supported standards of care. In a cancer care setting, this will often involve explicit acknowledgement that for both cancer patients and oncologists, low probability of success or lack of evidence of efficacy is not akin to quantitative futility. Awareness of this feature of cancer care is especially useful for the clinician who is not an oncologist, but works in a cancer care setting. Further, when such disagreements arise, the involved parties should consult the available facts in an effort to resolve the disagreement. In some cases, it may turn out that disagreement about quantitative futility is rooted in disagreement about other clinical facts, or about the relevant goal for making this determination. When this is the case, there is opportunity to resolve the disagreement about quantitative futility. When disagreements about quantitative futility cannot be resolved by appealing to the clinical facts, more difficult decisions may have to be made. For physicians who take themselves to have an obligation to refrain from offering or providing quantitatively futile interventions, the appropriate course of action is to offer only those medical tasks that are clinically indicated. Regardless of the provider's efforts to discuss solely indicated interventions, some patients or families might ask about or perhaps request inappropriate interventions. In instances such as this, the most appropriate strategy may be to refuse such requests and offer to transfer the patient's care to another physician or to another institution. Please see Table 2 for the combination of the original Gallagher/Holmes strategies with the additions (in bold) discussed in this paper.

\section{Conclusion}

Cases involving questions of medical futility can be especially challenging within a cancer care setting. However, by being explicit about the connections between particular interventions and goals of care, including non-curative goals of care, maintaining the distinction between quantitative and qualitative futility, and allowing for the possibility of reasonable disagreement about whether an intervention is quantifiably futile, clinicians, clinical ethicists, and patients/families are better able to resolve questions of medical futility within the cancer care environment.

\section{References}

1. Jecker NS, Pearlman RA (1992) Medical futility. Who decides? Arch Intern Med 152: 1140-1144.

2. Jecker NS, Schneiderman LJ (1993) Medical futility: the duty not to treat. Camb Q Healthc Ethics 2: 151-159.

3. Schneiderman LJ, Jecker NS, Jonsen AR (1990) Medical futility: its meaning and ethical implications. Ann Intern Med 112: 949-954.

4. Truog RD (2012) Medical futility. Georgia State University Law Review 25: 13 .

5. Veatch RM (2013) So-called futile care: The experience of the United States. Medical Futility: A Cross-National Study. Imperial College Press. London.

6. Jox RJ, Schaider A, Marckmann G, Borasio GD (2012) Medical futility at the end of life: the perspectives of intensive care and palliative care clinicians. J Med Ethics 38: 540-545.

7. Lantos JD, Singer PA, Walker RM, Gramelspacher GP, Shapiro GR, et al. (1989) The illusion of futility in clinical practice. Am J Med 87: 81-84.

8. Lo B (1995) Futile interventions. Resolving ethical dilemmas: A guide for clinicians. Baltimore: Williams \& Wilkins, 73-81.

9. Youngner SJ (1988) Who defines futility? JAMA 260: 2094-2095.

10. Youngner SJ (1990) Futility in context. JAMA 264: 1295-1296.

11. Callahan D (1991) Medical futility, medical necessity. The-problemwithout-a-name. Hastings Cent Rep 21: 30-35.

12. Gallagher CM, Holmes RF (2012) Handling cases of 'medical futility'. HEC Forum 24: 91-98.

13. Gallagher CM, Holmes RF (2011) Retrospective Review of Medical Futility and Ethics Consultations at MD Anderson Cancer Center. Journal of Clinical Research \& Bioethics 2:115.

14. Chen EX, Tannock IF (2004) Risks and benefits of phase 1 clinical trials evaluating new anticancer agents: a case for more innovation. JAMA 292: 2150-2151.

15. Roberts TG Jr, Goulart BH, Squitieri L, Stallings SC, Halpern EF, et al. (2004) Trends in the risks and benefits to patients with cancer participating in phase 1 clinical trials. JAMA 292: 2130-2140.

16. Voelker R (1998) NO survival advantage. Jama 280: 873-873.

17. National Institute of Health (2008) FAQ: Clinical Trials.gov-Clinical Trial Phases.

18. Albert S, Ashforth BE, Dutton JE (2000) Organizational identity and identification: Charting new waters and building new bridges. Academy of management Review 25: 13-17.

19. Meropol NJ, Weinfurt KP, Burnett CB, Balshem A, Benson AB, et al. (2003) Perceptions of Patients and Physicians Regarding Phase I Cancer Clinical Trials: Implications for Physician-Patient Communication. Journal of Clinical Oncology 21: 2589-2596.

20. Lenrow DA (2006) Treating Physician as Researcher: Is Assuming This Dual Role a Violation of the Nuremberg Code, The Temp J Sci Tech \& Envtl L 25: 15.

21. Kamal AH, Peppercorn JM (2013) The generalizability paradox within palliative care clinical trials. Ann Palliat Med 2: 101-104.

22. Smith TJ, Temin S, Alesi ER, Abernethy AP, Balboni TA, et al. (2012) American Society of Clinical Oncology provisional clinical opinion: the 
Citation: Gallagher CM (2016) Medical Futility in Cancer Care: Distinct Challenges and Action Strategies. J Clin Res Bioeth 7: 269. doi: 10.4172/2155-9627.1000269

Page 6 of 6

integration of palliative care into standard oncology care. J Clin Oncol 30: 880-887.

23. Yennurajalingam S, Kang JH, Cheng HY, Chisholm GB, Kwon JH, et al (2013) Characteristics of advanced cancer patients with cancer-related fatigue enrolled in clinical trials and patients referred to outpatient palliative care clinics. Journal of pain and symptom management 45 : $534-541$. 